\title{
Impact of Specifically Adsorbing Anions on the Electroless Growth of Gold Nanotubes
}

\author{
Falk Muench, Cornelia Neetzel, Stefan Lauterbach, \\ Hans-Joachim Kleebe, and Wolfgang Ensinger \\ Department of Materials and Geoscience, Technische Universität Darmstadt, Petersenstraße 23, 64287 Darmstadt, Germany \\ Correspondence should be addressed to Falk Muench, muench@ca.tu-darmstadt.de
}

Received 14 July 2011; Accepted 28 October 2011

Academic Editor: Renzhi Ma

Copyright (C) 2012 Falk Muench et al. This is an open access article distributed under the Creative Commons Attribution License, which permits unrestricted use, distribution, and reproduction in any medium, provided the original work is properly cited.

\begin{abstract}
Electroless metal deposition on nanochannel-containing templates is a versatile route towards metal nanotubes and nanowires if the plating reaction can be sufficiently controlled. In this study, disulfitoaurate-formaldehyde-based gold plating baths were modified by the addition of halides, pseudohalides, and EDTA. The introduction of specifically adsorbing anions strongly affected the heterogeneously autocatalyzed plating reaction and allowed the regulation of the reaction rate and the product morphology. The new plating baths showed enhanced stability and allowed the synthesis of homogeneous nanotubes of high aspect ratios ( $>150)$ in $30 \mu \mathrm{m}$ thick ion track-etched polymer templates. Depending on the reaction conditions, solid and porous structures consisting of gold nanoparticles of differing size and shape were accessible. The presented strategy offers adapted gold thin films, nanotubes, and nanowires for applications in catalysis or sensing.
\end{abstract}

\section{Introduction}

Nanotubes (NTs) are an important class of virtually onedimensional nanomaterials [1], which are deployed in various applications of high academical and practical interest. The versatility of NTs is supported by the broad range of materials from which they can be created. Besides carbon [2], NTs can be prepared from polymers [3, 4], metals [1, 4-8], and compounds such as oxides, sulfides, or nitrides [1] next to composites $[1,3]$. Among the presented materials, metal NTs exhibit a combination of unique physical and chemical properties such as electrical conductivity, plasmon resonance [5], catalytic activity $[6,7]$, and a rich surface chemistry involving adsorbates such as thiols $[7,8]$. Their superior properties lead to the implementation of Au NTs in a wide range of applications. For instance, they were effectively applied as biosensors [5], nanoreactors [6], supportless CO oxidation catalysts [7], and molecular sieves [8].

Usually, Au NTs are prepared according to a versatile template-based method introduced by Martin et al. [4, 7-10]. In this protocol, Au is electrolessly plated on ion track-etched polymer membranes. Electroless depositions are solutionbased reactions in which metal cations are selectively reduced on a substrate surface by a chemical agent [11]. Electroless Au plating leads to the development of polycrystalline films consisting of interconnected Au nanoparticles (NPs) $[9,10]$. If the metal film covers the nanochannel walls inside the template, Au NTs are formed.

While template preparation by ion track etching is well understood and ensures a high degree of control over the NT diameter, length, and shape [12], less effort has been devoted to the development of new electroless Au baths allowing to adjust the morphology of the plated Au thin films and NTs [6]. Preceding synthetic studies elucidated the effect of different parameters like the Au precursor concentration [9], the temperature [9], the $\mathrm{pH}$ value $[9,10]$, and the delayed introduction of the reducing agent [10] without changing the general bath composition. The results of these studies show relatively large Au NP sizes (typical average diameters lie between 30 and more than $100 \mathrm{~nm}$ ) and a limitation of the NP shape to globular morphologies $[9,10]$. However, control over the size and shape of metal NPs is of major nanotechnological importance since these parameters considerably affect properties such as catalytic selectivity and activity, optical absorption, and local field enhancement [13$15]$. 
As the presence of adsorbates strongly influences the growth of metal NPs [15], the introduction of adsorbing species is a straightforward measure to control both the deposition kinetics of electroless plating reactions and the morphology of the obtained products [6]. Halides are common reagents that readily adsorb specifically on noble metal surfaces [16] and show pronounced shape-directing effects in metal NP syntheses $[15,17,18]$. Thus, they are promising additives in the electroless fabrication of Au nanomaterials. In this study, we therefore examine the influence of halides and pseudohalides on the electroless synthesis of $\mathrm{Au}$ thin films and NTs and show how they can be used to optimize the obtained nanostructures towards a desired product.

\section{Experimental Procedure}

2.1. General, Chemicals. All glassware was cleaned with aqua regia prior to use, and all procedures were performed with purified water (Milli Q 18-M $\Omega$ water). The sensitization and plating solutions were prepared freshly, and the activation solution was stored dark and cold. The following chemicals were used without further purification: $\mathrm{AgNO}_{3}$ (Grüssing, p.a.), EDTA disodium salt dihydrate (Fluka, puriss. p.a.), ethanol (Brenntag, 99.5\%), formaldehyde solution 37\% in water, methanol stabilized (Grüssing, p.a.), KBr (Merck, suprapur), KI (Grüssing, 99.5\%), KSCN (Merck, cryst. pure), methanol (Sigma-Aldrich, laboratory reagent), $\mathrm{NaCl}$ (Merck, suprapur), $\mathrm{NaCN}$ (Merck, p.a.), $\mathrm{Na}_{2} \mathrm{SO}_{3}$ (Merck, p.a.), $\mathrm{NH}_{3} 33 \%$ in water (Merck, puriss.), $\mathrm{SnCl}_{2}$ dihydrate (Sigma-Aldrich, ACS reagent), sodium hydroxide solution $32 \%$ in water (Fluka, puriss. p.a.), and trifluoroacetic acid (Riedel-de Haën, >99\%). A commercial electroplating solution (El-Form Galvano Goldbad, Schütz Dental GmbH) was used as the Au source (15 g 99.9\% Au per liter, present as $\left.\left(\mathrm{NH}_{4}\right)_{3}\left[\mathrm{Au}\left(\mathrm{SO}_{3}\right)_{2}\right]\right)$.

2.2. Synthesis. Polycarbonate foils (Makrofol, Bayer MaterialScience $A G$, nominal thickness $30 \mu \mathrm{m}$ ) were irradiated with $\mathrm{Au}$ ions (energy: $11 \mathrm{MeV}$ per nucleon, fluence: $1 \cdot 10^{8}$ ions $\mathrm{cm}^{-2}$ ) at the Helmholtz centre for heavy ion research (GSI). Subsequently, they were irradiated with UV light in the presence of air ( $1 \mathrm{~h}$ per side, UV source provides 1.5 and $4 \mathrm{Wm}^{-2}$ in the ranges of $280 \mathrm{~nm}-320 \mathrm{~nm}$ and $320 \mathrm{~nm}-$ $400 \mathrm{~nm}$, resp.) and etched in stirred sodium hydroxide solution $\left(6 \mathrm{M}, 50^{\circ} \mathrm{C}\right.$, time depending on desired diameter, ranging from 100 to $200 \mathrm{~nm}$ ). The as-prepared templates were thoroughly washed with water and dried. Prior to electroless plating, the polycarbonate substrates were activated with Ag NPs according to a previously reported procedure [6] (for a description of the process, see Section 3.1 and Figure 1). After washing with ethanol and water, the freshly activated substrates were immersed in the electroless plating solutions. The $\mathrm{pH}$ of all plating solutions was adjusted to 9.3-9.4 with $\mathrm{NaOH}$ since moderately basic $\mathrm{pH}$ values favor dense nucleation, low reaction rates and small crystallite sizes, and hence tube formation [10]. Temperature was held constant at $8^{\circ} \mathrm{C}$ to ensure slow deposition necessary for formation of tubes of homogeneous wall thickness [9]. The

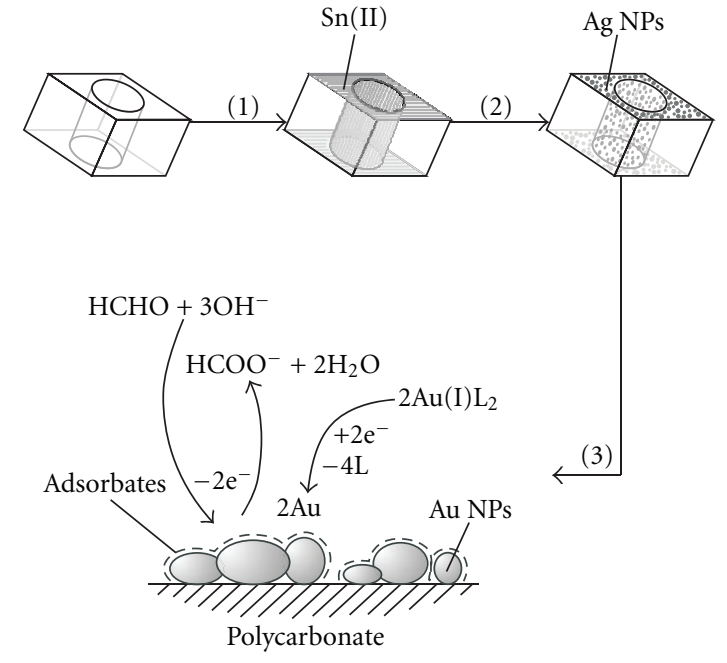

FIGURE 1: Scheme of the Au NT fabrication in ion-track-etched polymer templates. (1) Sensitization by anchoring $\mathrm{Sn}$ (II) ions on the template. (2) Activation by immersion in a $\mathrm{Ag}(\mathrm{I})$ solution, leading to the formation of Ag NPs on the inner and outer template surface. (3) Electroless Au plating using a $\mathrm{Au}(\mathrm{I})$ complex ( $\mathrm{L}=$ ligand) as the oxidizing and formaldehyde as the reducing agent. The deposition reaction is altered by specifically adsorbing additives.

Au plating baths contained $7.0 \mathrm{mM} \mathrm{Au}(\mathrm{I}), 125 \mathrm{mM} \mathrm{Na}_{2} \mathrm{SO}_{3}$, and $625 \mathrm{mM}$ formaldehyde. All additives have been initially applied in a concentration of $100 \mathrm{mM}$. In case of strong interference with the plating reaction, the concentrations have been reduced to the values stated in the text. In the cases of mixed addition of EDTA and salts containing specifically adsorbing anions, the concentration of EDTA was held constant at $100 \mathrm{mM}$ while the salt concentrations were varied. After the desired reaction time, the membranes were washed with water and dried.

2.3. Characterization. TEM (FEI CM 20 microscope (Netherlands), $200 \mathrm{kV}$ acceleration voltage, $\mathrm{LaB}_{6}$-cathode): the NTcontaining templates were embedded in Araldit 502 (polymerization at $60^{\circ} \mathrm{C}$ for $16 \mathrm{~h}$ ) and examined as ultrathin sections (70 nm thickness, Reichert-Jung Ultracut E ultramicrotome, DKK diamond knife). EDS (Oxford Model 6767 EDS-system (England)): in combination with TEM, EDS measurements were performed to confirm the composition of the obtained nanostructures. A representative EDS spectrum is shown in Figure 6. SEM (JEOL JSM-7401F, 5$10 \mathrm{kV}$ acceleration voltage): prior to the measurement, the template was removed with dichloromethane. The freed metal structures were collected on silicon wafer pieces sputter-coated with Au.

\section{Results and Discussion}

3.1. Synthetic Strategy. Generally, electroless plating reactions are not catalyzed by pristine polymer surfaces [11]. Therefore, polymer substrates have to be modified to initiate 
metal deposition. In case of ion-track-etched polymer templates, catalytic activity is usually established by covering the substrate with Ag NPs $[4,6,9,10]$. This process involves two steps. First, the template is immersed in an $\mathrm{SnCl}_{2}$ solution, leading to the formation of surface complexes between $\mathrm{Sn}$ (II) and polar groups of the polymer (sensitization, see step (1) in Figure 1). After washing off the excess of unbound $\mathrm{SnCl}_{2}$, the template is dipped in an $\left[\mathrm{Ag}\left(\mathrm{NH}_{3}\right)_{2}\right] \mathrm{NO}_{3}$ solution. The $\mathrm{Ag}(\mathrm{I})$ oxidizes $\mathrm{Sn}$ (II) forming $\mathrm{Sn}(\mathrm{IV})$, while $\mathrm{Ag}(\mathrm{I})$ is reduced to elemental Ag. This leads to the precipitation of Ag NPs on the template surface (activation, see step (2) in Figure 1) $[9,10]$. After activation, the template membranes are washed and finally immersed in the plating solution (step (3) in Figure 1). Electroless plating baths applied in the synthesis of Au NTs contain $\left[\mathrm{Au}\left(\mathrm{SO}_{3}\right)_{2}\right]^{3-}$ as the stabilized $\mathrm{Au}(\mathrm{I})$ source and formaldehyde as the reducing agent [7-10]. During the heterogeneously autocatalyzed plating reaction, formaldehyde is oxidized to formate, while $\mathrm{Au}(\mathrm{I})$ is reduced to elemental $\mathrm{Au}$ [9]. A side reaction is the cementation of $\mathrm{Au}$ on the less noble Ag introduced during activation (1) [9]:

$$
\mathrm{Ag}+\mathrm{Au}(\mathrm{I}) \longrightarrow \mathrm{Au}+\mathrm{Ag}(\mathrm{I}) .
$$

Heterogeneous reactions severely respond to interfacial changes. For instance, Pt catalysts are poisoned by strongly bonding species such as CO [19], whereas the adsorption of chiral molecules such as cinchona alkaloids can lead to enhanced reaction rates and asymmetric induction on them [20]. Adsorbing species such as cationic surfactants, halides, and silver salts play, a major role in the wetchemical synthesis of anisotropic Au NPs [21]. Analogous to colloidal approaches, electroless plating is based on metal NP formation by the reduction of dissolved cationic precursors. Correspondingly, the presence of adsorbates strongly affects the reaction kinetics and the shape of the evolving nanostructures $[6,21]$. Furthermore, adsorbate-forming additives can improve the stability of plating baths towards homogeneous nucleation [6]. This issue is an important quality criterion in electroless plating. If the metal reduction is not limited to the substrate surface, homogeneous nucleation leads to the formation of metal nuclei in the bulk solution. Continued nucleation and the growth of existing particles quickens the depletion of the plating solution and gives rise to undesired metal precipitation. In addition, homogeneous nucleation negatively affects plating rates and often leads to deteriorated metal films [22]. Applying the outlined concept, we investigated the influence of different halides and pseudohalides (chloride, bromide, iodide, cyanide, and thiocyanate) on the electroless growth of Au NTs. Next to adsorbing anions, the universal ligand EDTA was tested as a complementary complexing agent. In contrast to the reactive components $\mathrm{Au}(\mathrm{I})$ and formaldehyde, the applied additives are not consumed during the plating reaction (step (3) in Figure 1).

\subsection{Isolated Effect of Additives}

3.2.1. EDTA. Due to its relatively low-binding strength towards low-valent metal cations and its competition with sulfite as a soft sulfur-containing ligand well suited for the coordination of the soft Lewis acid $\mathrm{Au}(\mathrm{I})$, the presence of EDTA should not lead to a pronounced additional stabilization of the oxidizing component $\mathrm{Au}(\mathrm{I})$. Accordingly, the addition of EDTA does not alter the electroless deposition reaction to a relevant degree.

Similar product morphologies and reaction speeds were obtained for the plating baths containing sulfite and sulfite next to EDTA (Figures 2(a)-2(b)). In both cases, the Au NTs were found to be composed of granular NPs of approximately 15-20 nm size. Also, the Au NTs showed fragmentation when the polycarbonate template was removed. With increasing distance to their openings, the tubes become crumbly and finally break after some micrometers (Figure 2(b), see arrow). Longer fragments were found in the presence of EDTA, revealing slightly improved NT formation. The inhomogeneous deposition of $\mathrm{Au}$ alongside the template nanochannels is caused by the relatively high-deposition rate [9]. While the reactive species of the plating bath are consumed on the whole inner template surface, mass transport from the bulk solution is limited by the narrow tube openings. This leads to reagent depletion and reduced plating rates inside the NTs.

3.2.2. Halides. The addition of $100 \mathrm{mM}$ chloride or bromide lead to similar changes in Au plating concerning the product morphology and the deposition rate. At the beginning of the plating reaction, a relatively quick color-shift of the activated templates from brownish to pink occurred, which was attributed to Au cementation (1). The electroless deposition of larger amounts of $\mathrm{Au}$, which was indicated by the development of a dark purple or bluish color was decelerated in the presence of chloride and bromide. While chloride leads to a moderate reduction of the deposition rate, bromide caused a drastic deceleration. In both cases, the development of continuous Au films and well-defined Au NTs was hampered. Instead, the growth of separated NPs was observed (Figures 3(a)-3(b), arrow indicates an isolated particle). Compared to chloride, bromide had a more pronounced effect on the product morphology. While in case of chloride tube fragments were found after a reaction of one day (Figure 3(a)), no NTs were observed in the case of the addition of $100 \mathrm{mM}$ bromide even after a deposition time of 10 days (Figure 3(b)). However, the formation of fine NTs of approximately $100 \mathrm{~nm}$ diameter was confirmed by TEM (Figure 3(c)). The NTs consist of particles of $7.7 \pm 1.5 \mathrm{~nm}$ size. As no continuous Au film growth was observed in this experiment, the tubular structures are probably composed of Au NPs stemming from the Ag conversion (1).

The deceleration of electroless plating in the presence of halides can be explained by partial poisoning of the $\mathrm{Au}$ surface due to adsorbate formation. Compared to chloride, the heavier halide bromide has a higher polarizability and interacts stronger with Au surfaces [16]. As a result, bromide leads to a higher surface coverage at identical solution concentration [16]. With increasing blockage of the reactive sites on the Au surface, the plating reaction should be increasingly constrained. In accordance with this expectation, bromide has a stronger effect on the electroless Au deposition. 


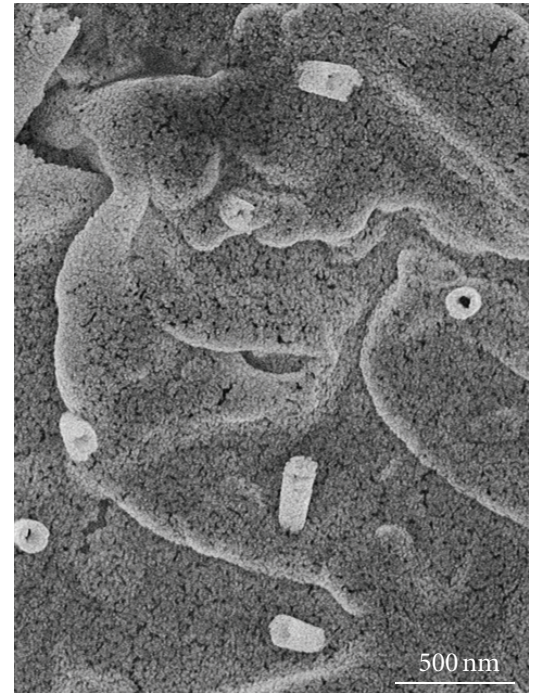

(a)

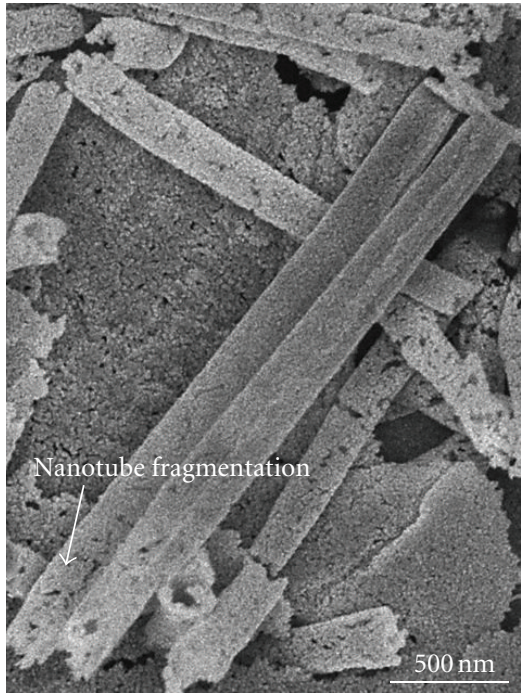

(b)

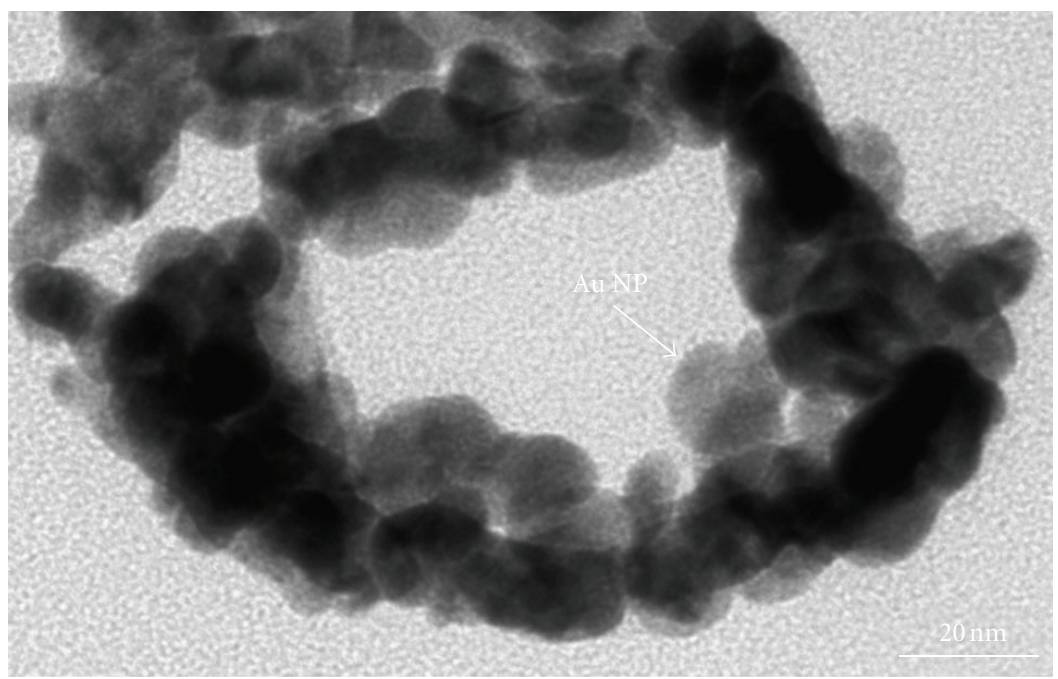

(c)

FIgURE 2: Electron microscopy images of Au NTs grown without (a) and with (b-c) addition of EDTA. (a) and (b) comprise SEM images of template freed Au NTs, (c) a TEM image of a microtomed Au NT (c). Both depositions took place for $21 \mathrm{~h}$ in templates with nanochannels of approximately $200 \mathrm{~nm}$ entrance diameter.

Interestingly, the trend did not extend to the heaviest homologue iodide. In contrast to the expectation of an efficient reduction of surface activity, electroless plating baths containing $100 \mathrm{mM}$ iodide quickly decomposed to form $\mathrm{Au}$ suspensions. Even extremely reduced iodide concentrations below $1 \mathrm{mM}$ accelerated both the homogeneous and heterogeneous gold reduction and were thus detrimental to NT formation. The opposing behavior of iodide compared to its lighter homologues is surprising, but highly reproducible. An increase of the speed of electrochemical reactions on metal surfaces with anion adlayers is a known phenomenon. It can be explained by the lowering of the activation energy of intermediate species by the adsorbed anions [16]. In the case of accelerated Au plating, either formaldehyde oxidation or the integration of $\mathrm{Au}$ atoms in the evolving $\mathrm{Au}$ film may be supported by iodide. The responsible mechanism is evidently ineffective in the case of chloride or bromide addition. Another example for an unexpected change of surface reactivity in the presence of iodide is the anodic dissolution of Pd [23]. In contrast to the corrosion-enhancing behavior of chloride, which depends on the bulk chloride concentration, even a monolayer of iodide was sufficient to increase the Pd dissolution rate by a factor of nearly 200 [23].

The formation of halide complexes as an explanation for the altered deposition kinetics is unprobable due to the strong tendency of $\mathrm{Au}(\mathrm{I})$ to form sulfito complexes. This is indicated by the significantly more negative standard potential of the disulfitoaurate complex compared to the analogous bromo- and iodoaurate complexes:

$$
\left[\mathrm{AuBr}_{2}\right]^{-}+\mathrm{e}^{-} \longrightarrow \mathrm{Au}+2 \mathrm{Br}^{-} \quad E=0.96 \mathrm{~V}[24]
$$




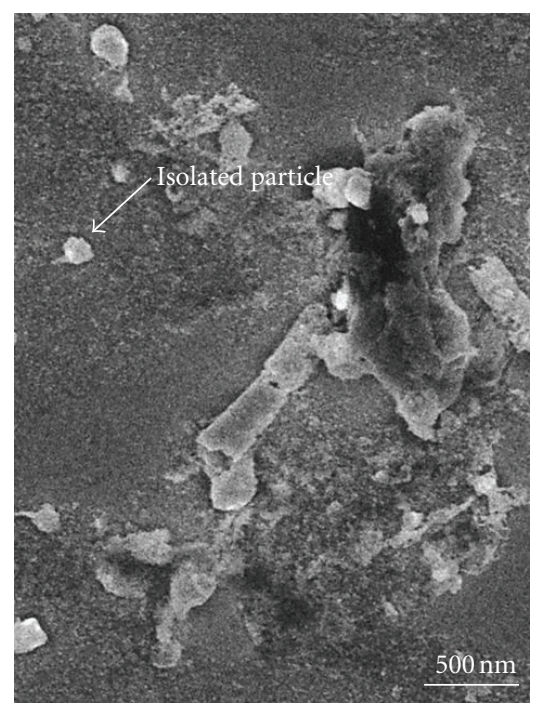

(a)

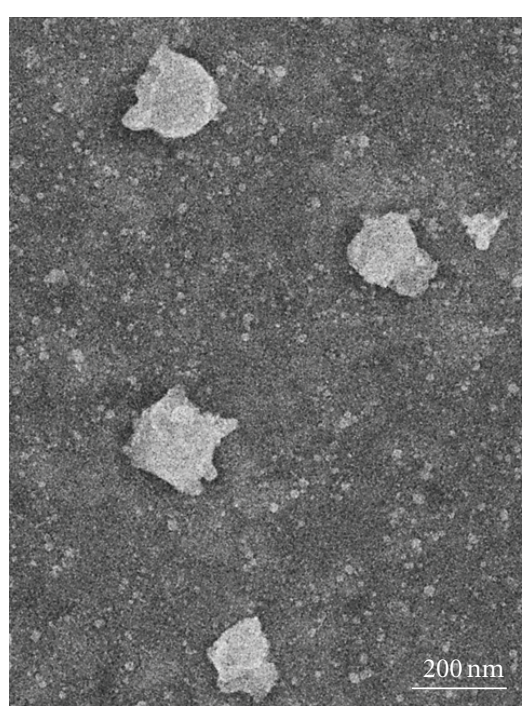

(b)

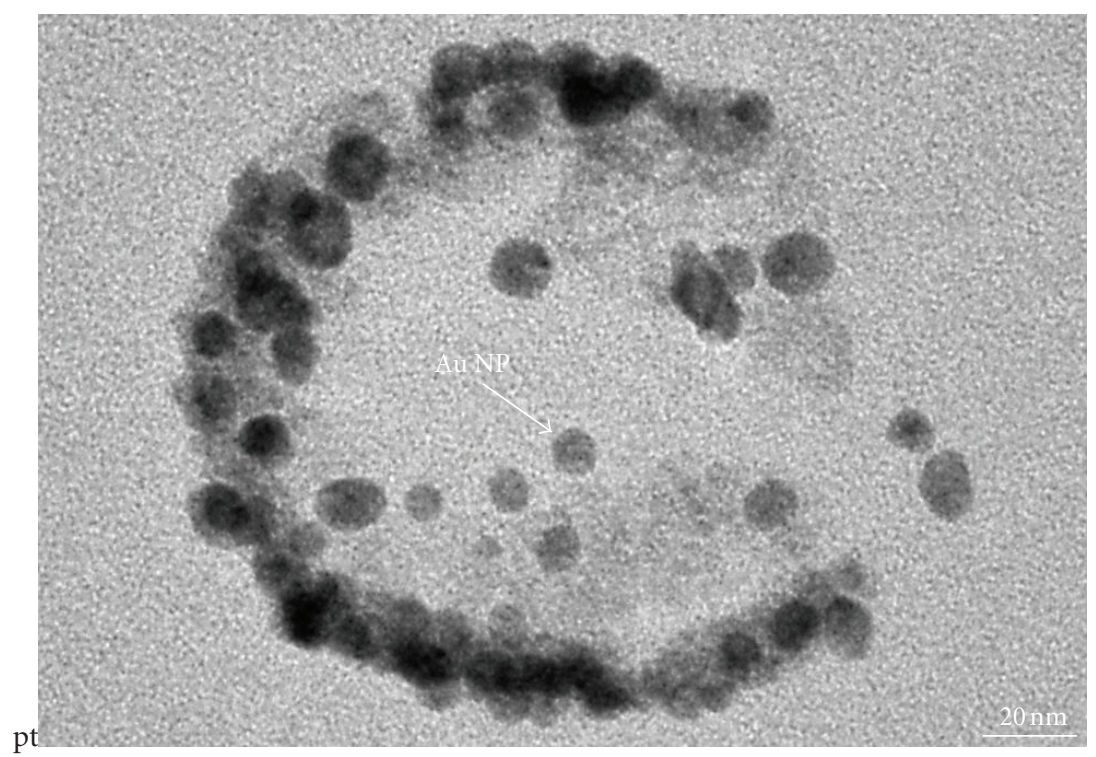

(c)

Figure 3: Electron microscopy images of Au nanostructures grown with the addition of $100 \mathrm{mM}$ chloride (a) or $100 \mathrm{mM}$ bromide (b-c). (a) and (b) comprise SEM images of template-freed Au nanostructures and (c) a TEM image of a microtomed Au NT. The reaction time was $21 \mathrm{~h}$ for the samples shown in (a) and (c) and 10 days for the sample shown in (b).

$$
\left[\mathrm{AuI}_{2}\right]^{-}+\mathrm{e}^{-} \longrightarrow \mathrm{Au}+2 \mathrm{I}^{-} \quad E=0.58 \mathrm{~V}[24]
$$

$$
\left[\mathrm{Au}\left(\mathrm{SO}_{3}\right)_{2}\right]^{3-}+\mathrm{e}^{-} \longrightarrow \mathrm{Au}+2 \mathrm{SO}_{3}{ }^{2-} \quad E=0.116 \mathrm{~V}[25] .
$$

If the lowered standard potential of a metal complex is ascribed to the reduced concentration of free metal ions (in this case solvated $\mathrm{Au}(\mathrm{I})$ ), its complex formation constant can be calculated on basis of the Nernst equation. The difference between the standard potentials $E_{n}$ of two complexes is related to the ratio of their complex formation constants
$K_{n}$ in the following way ( $z$ : charge, $F$ : Faraday constant, $R$ : universal gas constant, $T$ : temperature):

$$
\frac{K_{1}}{K_{2}}=\mathrm{e}^{\left(E_{2}-E_{1}\right) z F / R T} .
$$

With $\mathrm{Au}(\mathrm{I})$ as a monovalent cation $(z=1)$ and $T=298 \mathrm{~K}$, a potential difference of $59 \mathrm{mV}$ corresponds to $K$ values deviated by one order of magnitude. The application of (5) to (2)-(4) leads to $K$ ratios between the sulfito complex and the bromo- and iodo complexes of $1.9 \cdot 10^{14}$ and $7.0 \cdot 10^{7}$, respectively. Due to the markedly stronger complexation of $\mathrm{Au}(\mathrm{I})$ by sulfite, it can be stated clearly that even the heavy 


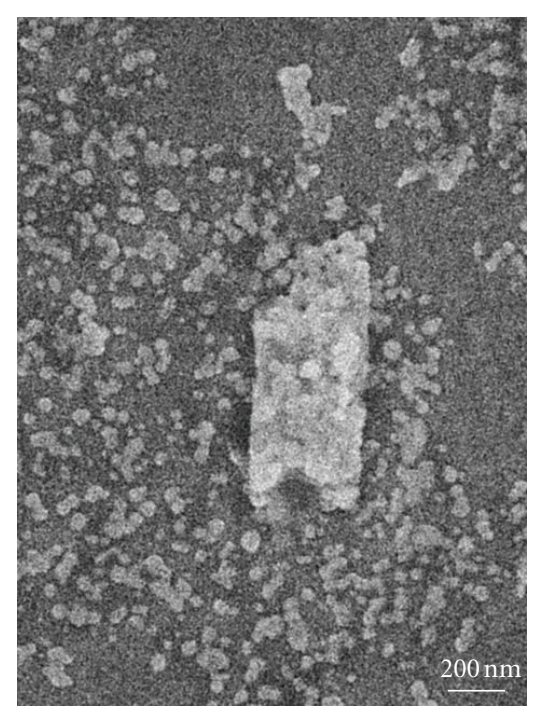

(a)

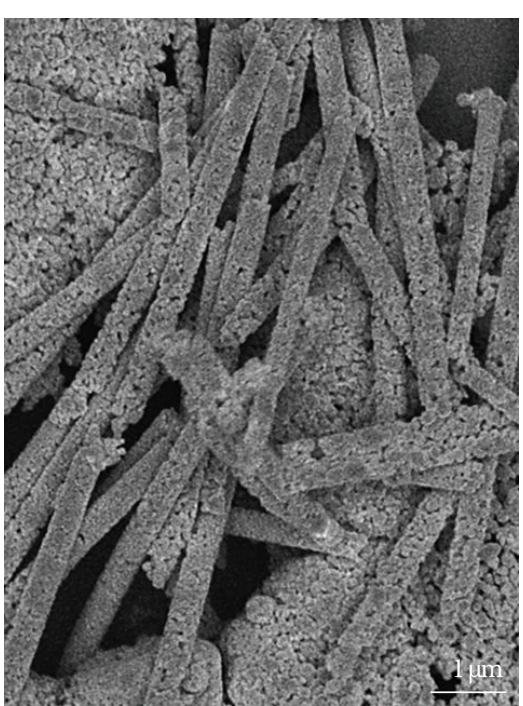

(b)

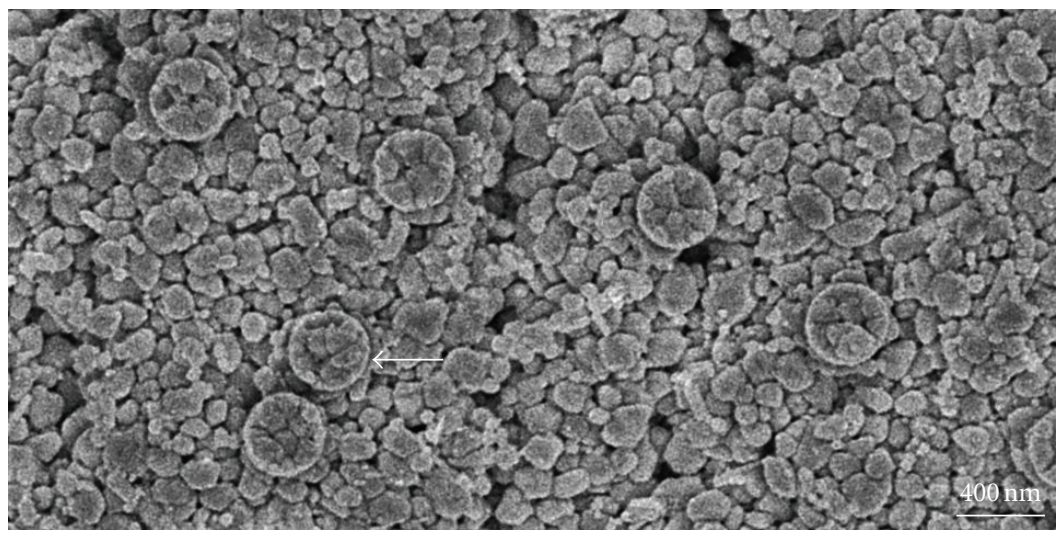

(c)

FiguRE 4: SEM images of template-freed Au nanostructures grown in the presence of $100 \mathrm{mM}$ thiocyanate. (a) Au structures yielded after a reaction time of $21 \mathrm{~h}$. (b) Au NTs obtained after a deposition time of 4 days. (c) Magnified image of the Au surface film of the sample shown in (b), the arrow marks a nanowire stump.

halides bromide and iodide do not significantly contribute as ligands under the given experimental conditions (similar concentrations of sulfite and halides, no excess of $\mathrm{Au}(\mathrm{I}))$.

3.2.3. Pseudohalides. The presence of $100 \mathrm{mM}$ cyanide completely stopped the electroless deposition reaction. One reason for this behaviour is the formation of the strongly stabilized dicyanoaurate complex. Its standard potential (6) is significantly negative-shifted compared to the disulfitoaurate complex (3):

$$
\left[\mathrm{Au}(\mathrm{CN})_{2}\right]^{-}+\mathrm{e}^{-} \longrightarrow \mathrm{Au}+2 \mathrm{CN}^{-} \quad E=-0.60 \mathrm{~V}[24]
$$

The high thermodynamic driving force towards dicyanoaurate even allows etching of Au by molecular oxygen [24], a technique which is used in gold extraction as [25]well as in the shape modification of Au nanomaterials [26]. In our plating bath, formaldehyde with a standard potential of $-0.927 \mathrm{~V}[27]$ could still reduce $\left[\mathrm{Au}(\mathrm{CN})_{2}\right]^{-}$. But in addition to the thermodynamic stabilization of $\mathrm{Au}(\mathrm{I})$, cyanide is known to poison Au surfaces in electroless plating [28].

$$
\left[\mathrm{Au}(\mathrm{SCN})_{2}\right]^{-}+\mathrm{e}^{-} \longrightarrow \mathrm{Au}+2 \mathrm{SCN}^{-} \quad E=0.69 \mathrm{~V}[24] .
$$

In contrast to cyanide, thiocyanate cannot compete with sulfite as a ligand (7). Application of (5) results in a sulfite complex formation constant, which is $5.1 \cdot 10^{9}$ times higher than the corresponding value for thiocyanate. Therefore, the change of the $\mathrm{Au}$ deposition induced by thiocyanate can be related to its adsorption behaviour. Its presence has a considerable effect on the Au film growth, leading to a reduced plating rate, improved suppression of homogeneous $\mathrm{Au}$ precipitation, and enlarged Au crystallites (Figure 4). During $21 \mathrm{~h}$ of deposition, a granular Au film and fragile NTs develop (Figure 4(a)). After extended reaction times, a coarsening of the Au NPs occurs and Au nanowires are formed due to the complete filling of the template nanochannels (Figures 4(b)-4(c)). The rough Au films consist of 

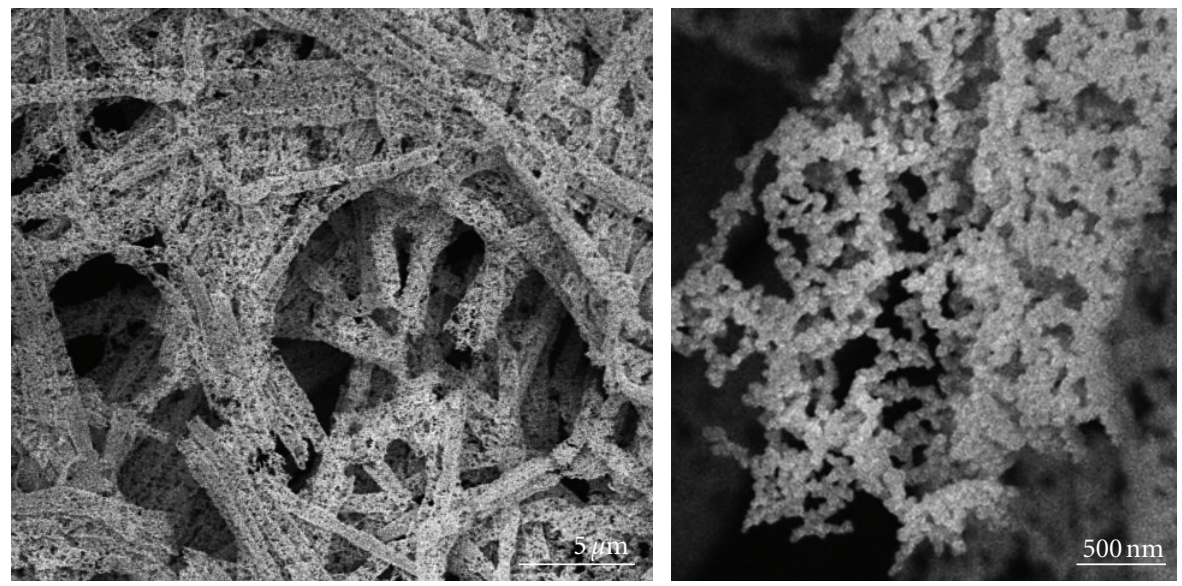

(a)
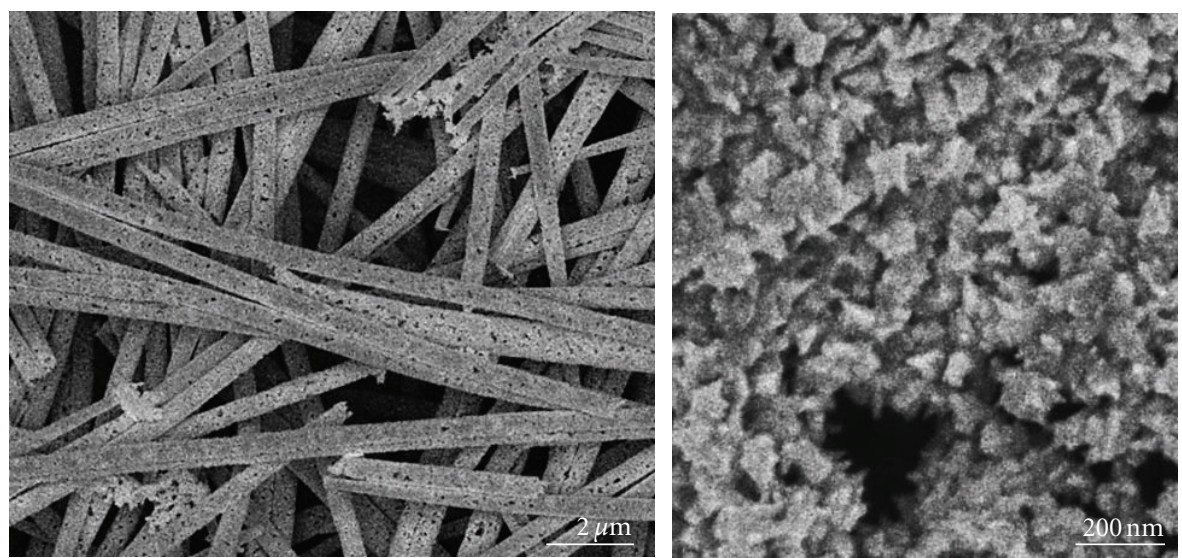

(b)
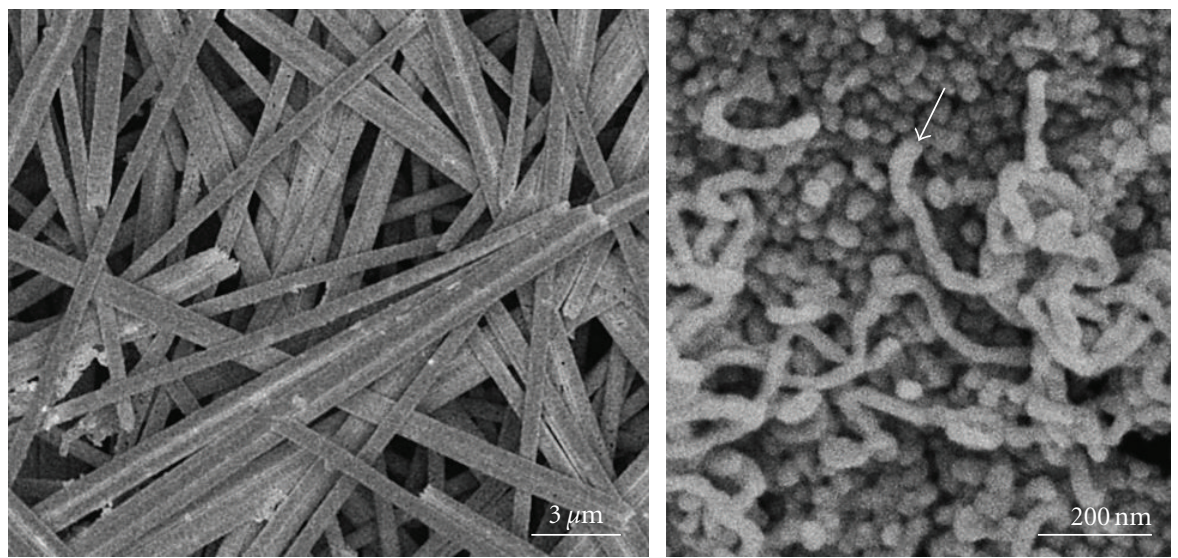

(c)

FIGURE 5: SEM images of template-freed Au nanostructures obtained with combined addition of EDTA and (a) $20 \mathrm{mM}$ chloride, (b) $0.25 \mathrm{mM}$ bromide, and (c) $0.25 \mathrm{mM}$ iodide. The images on the right show magnified features of the obtained structures (porous Au NTs in (a), Au surface film in (b), and nanowires emerging from the surface film in (c)).

interconnected, edgy grains, and many small gaps remain between the particles (Figure 4(c)). Therefore, thiocyanate is a suitable additive for the electroless synthesis of roughened Au nanostructures. Surface roughness is an important factor considering implementation in catalysis, superhydrophobicity, and sensing. For instance, rough Au films can be applied as surface enhanced Raman spectroscopy (SERS) substrates [29] or in adsorbate sensing [30].

3.3. Combined Effect of EDTA and (Pseudo)halides. The effects of halides on the plating reaction are strongly altered 
by the addition of EDTA. In all depositions, significantly reduced plating rates and morphology changes were observed. According to the halide adsorption tendency, bromide and iodide have the most intense effect on the electroless plating. All halides allow the synthesis of high aspect ratio $\mathrm{Au}$ NTs with homogeneous wall structure (Figure 5). Also the reduced reaction rates correlate with increased bath stabilities. In all mixed EDTA-halide experiments, homogeneous nucleation could be successfully suppressed. The $\mathrm{Au}$ NTs obtained with EDTA and thiocyanate were not coherent and completely disintegrated to form NP suspensions during washing of the NT-containing polycarbonate membranes. Some NP loss also occurred in the case of Au films grown in the presence of chloride and bromide.

In case of chloride, the initially formed Au NPs are only partly connected, leading to porous Au films and NTs in the early stages of the plating reaction. In Figure 5(a), the product obtained after a reaction time of one day is shown, revealing a particle size of approximately $30 \mathrm{~nm}$. In later stages, closed Au films and NTs are obtained. Removing the template from a porous sample leads to the formation of a spongy Au NT network (Figure 5(a)). This structure is interesting because of its hierarchical porosity with macropores defined by the tube interiors and mesoporous tube walls. Similar Ag structures have been implemented as effective substrates for surface-enhanced Raman scattering (SERS) [31]. Also, porous metal NTs are a promising class of unsupported heterogeneous catalysts [32].

In the EDTA-bromide system, grain size is tunable by adjusting the bromide concentration. A bromide concentration of $2.0 \mathrm{mM}$ leads to the formation of small globular particles of less than $10 \mathrm{~nm}$ size, approaching the product morphology obtained in the case of EDTA-free bromide baths (see Figures 3(b)-3(c)). At a concentration of $0.25 \mathrm{mM}$ and a reaction time of 8 days, the Au nanostructures are composed of sharp and interconnected grains with a size of few tens of nm (see right image in Figure 5(b)). Under these conditions, robust Au NTs are formed. Since metal NPs with high curvatures produce high field enhancements [21, 33], the obtained spiky Au films and NTs are interesting for SERS applications. In a seed-mediated approach, similar Au NP morphologies (branched and star-like) were yielded by the reduction of $\mathrm{HAuCl}_{4}$ in the presence of cetyltrimethylammonium bromide (CTAB) and optionally traces of $\mathrm{AgNO}_{3}$ [34]. Another solution-based route towards multibranched $\mathrm{Au}$ NPs is the reduction of $\mathrm{HAuCl}_{4}$ with hydrazine in the presence of polyvinylpyrrolidone. These structures have been implemented as effective SERS substrates [33].

Like in the experiments performed without EDTA, high iodide concentrations increase the deposition rate and the susceptibility to homogeneous nucleation. However, low iodide contents in combination with EDTA delay the plating reaction and suppress bath decomposition. Figure 5(c) shows the product obtained by the addition of $0.25 \mathrm{mM}$ iodide next to $100 \mathrm{mM}$ EDTA after a reaction time of two days. Next to nonfragmenting Au NTs, fibrous deposits are found on top of the solution-sided face of the Au surface film. The nanowires originate from single NPs of the surface films (see arrow in Figure 5(c)) and are even found at a

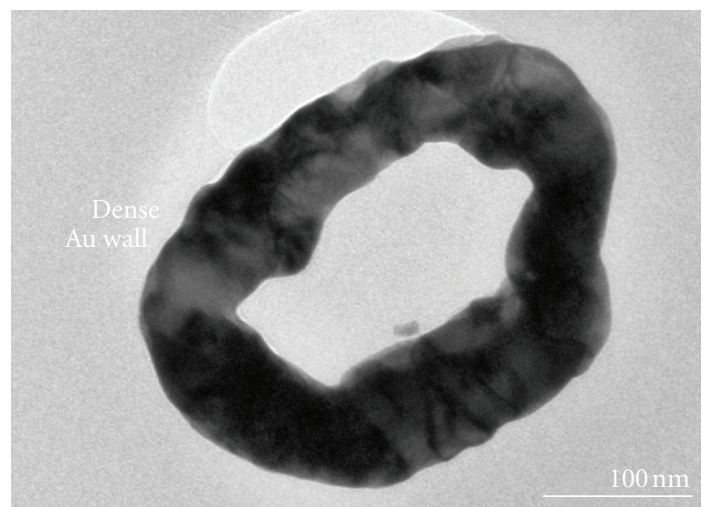

(a)

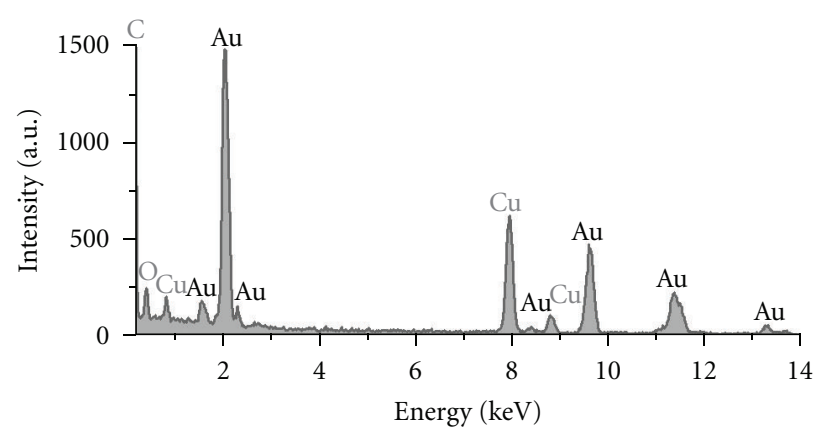

(b)

Figure 6: (a) TEM image of a microtomed sample of the Au NTs grown in the presence of $0.25 \mathrm{mM}$ iodide and $100 \mathrm{mM}$ EDTA. (b) Representative EDS spectrum of the Au nanostructures. The $\mathrm{C}$ and $\mathrm{O}$ signals are due to the polymer matrix and the $\mathrm{Cu}$ signals due to the TEM grid, respectively.

drastically reduced iodide concentration of $25 \mu \mathrm{M}$. The EDTA-iodide system yields NT walls composed of densely interconnected Au NPs (Figure 6(a)). By EDS, no significant $\mathrm{Ag}$ residues can be detected (Figure 6(b)). The obtained $\mathrm{Au}$ NTs are very robust and endure the template removing process without major deformation or fragmentation. A high mechanical strength of metal NTs allows the fabrication of free-standing NT arrays and increases the resistance of the nanostructures under stress. For instance, a quick loss of NPs would decrease the lifetime of metal NT catalysts. In addition, the nanotube structure affects its electrical conductivity. These factors are important for applications in catalysis [35, 36], nanocircuitry [37], plasmonic biosensing, or the nanoprobing of living cells [5].

\section{Conclusion}

Specifically adsorbing anions strongly affect the deposition kinetics and the product morphology in the disulfitoaurateformaldehyde plating system. Especially the heavy halides bromide and iodide with their high affinity towards $\mathrm{Au}$ surfaces show pronounced effects even in catalytic concentrations. This observation is in accordance with the high sensitivity of colloidal Au crystal growth towards the presence of specifically adsorbing anions $[17,18,21]$. Because of 
the increased bath stabilities and the improved plating behaviour, the addition of (pseudo)halides and optionally EDTA to disulfitoaurate-formaldehyde-based electroless plating baths is a facile route towards $\mathrm{Au}$ thin films and derived nanomaterials of variable morphology. Significantly reduced plating rates allow to cover substrates with complex and badly accessible surfaces with homogeneous Au NP films. In contrast to the standard plating procedure, sophisticated structures such as homogeneous and stable Au NTs of high aspect ratio could be synthesized using the refined plating procedures. With maximum tube entrance diameters between 150 and $200 \mathrm{~nm}$, the presented NTs approach aspect ratios of more than 150 . The stable Au NTs could be freed from the template without fragmentation, allowing straightforward processing and application of the nanostructures for example, in heterogeneous catalysis or sensing.

\section{Acknowledgments}

The authors thank Professor Reinhard Neumann and Dr. Christina Trautmann (GSI Helmholtz Centre for Heavy Ion Research) for support with the template preparation and for the possibility to use the SEM of the materials research group. Thanks are given to Ulrike Kunz for sample preparation and TEM imaging. The supply of gold solution by Schütz Dental $\mathrm{GmbH}$ is gratefully acknowledged.

\section{References}

[1] C. N. R. Rao and A. Govindaraj, "Synthesis of inorganic nanotubes," Advanced Materials, vol. 21, no. 42, pp. 4208-4233, 2009.

[2] D. Vairavapandian, P. Vichchulada, and M. D. Lay, "Preparation and modification of carbon nanotubes: review of recent advances and applications in catalysis and sensing," Analytica Chimica Acta, vol. 626, no. 2, pp. 119-129, 2008.

[3] J. Martín and C. Mijangos, "Tailored polymer-based nanofibers and nanotubes by means of different infiltration methods into alumina nanopores," Langmuir, vol. 25, no. 2, pp. 1181-1187, 2009.

[4] C. R. Martin, "Nanomaterials: a membrane-based synthetic approach," Science, vol. 266, no. 5193, pp. 1961-1966, 1994.

[5] J. McPhillips, A. Murphy, M. P. Jonsson et al., "High-performance biosensing using arrays of plasmonic nanotubes," ACS Nano, vol. 4, no. 4, pp. 2210-2216, 2010.

[6] F. Muench, U. Kunz, C. Neetzel, S. Lauterbach, H. J. Kleebe, and W. Ensinger, "4-(dimethylamino)pyridine as a powerful auxiliary reagent in the electroless synthesis of gold nanotubes," Langmuir, vol. 27, no. 1, pp. 430-435, 2011.

[7] M. A. Sanchez-Castillo, C. Couto, W. B. Kim, and J. A. Dumesic, "Gold-nanotube membranes for the oxidation of CO at gas-water interfaces," Angewandte Chemie-International Edition, vol. 43, no. 9, pp. 1140-1142, 2004.

[8] S. Yu, S. B. Lee, M. Kang, and C. R. Martin, "Size-based protein separations in poly(ethylene glycol)-derivatized gold nanotubule membranes," Nano Letters, vol. 1, no. 9, pp. 495-498, 2001.

[9] R. J. Gilliam, S. J. Thorpe, and D. W. Kirk, "A nucleation and growth study of gold nanowires and nanotubes in polymeric membranes," Journal of Applied Electrochemistry, vol. 37, no. 2, pp. 233-239, 2007.
[10] M. De Leo, F. C. Pereira, L. M. Moretto, P. Scopece, S. Polizzi, and P. Ugo, "Towards a better understanding of gold electroless deposition in track-etched templates," Chemistry of Materials, vol. 19, no. 24, pp. 5955-5964, 2007.

[11] I. A. Abu-Isa, "Metal plating of polymeric surfaces," PolymerPlast Technol Eng, vol. 2, no. 1, pp. 29-65, 1973.

[12] P. Apel, "Track etching technique in membrane technology," Radiation Measurements, vol. 34, no. 1-6, pp. 559-566, 2001.

[13] I. Lee, F. Delbecq, R. Morales, M. A. Albiter, and F. Zaera, "Tuning selectivity in catalysis by controlling particle shape," Nature Materials, vol. 8, no. 2, pp. 132-138, 2009.

[14] T. K. Sau, A. L. Rogach, F. Jäckel, T. A. Klar, and J. Feldmann, "Properties and applications of colloidal nonspherical noble metal nanoparticles," Advanced Materials, vol. 22, no. 16, pp. 1805-1825, 2010.

[15] Y. Xia, Y. Xiong, B. Lim, and S. E. Skrabalak, "Shapecontrolled synthesis of metal nanocrystals: simple chemistry meets complex physics?" Angewandte Chemie-International Edition, vol. 48, no. 1, pp. 60-103, 2009.

[16] O. M. Magnussen, "Ordered anion adlayers on metal electrode surfaces,” Chemical Reviews, vol. 102, no. 3, pp. 679-725, 2002.

[17] T. H. Ha, H. J. Koo, and B. H. Chung, "Shape-controlled syntheses of gold nanoprisms and nanorods influenced by specific adsorption of halide ions," Journal of Physical Chemistry C, vol. 111, no. 3, pp. 1123-1130, 2007.

[18] D. K. Smith, N. R. Miller, and B. A. Korgel, "Iodide in CTAB prevents gold nanorod formation," Langmuir, vol. 25, no. 16, pp. 9518-9524, 2009.

[19] H. Siwek, M. Łukaszewski, and A. Czerwiński, "Electrochemical study on the adsorption of carbon oxides and oxidation of their adsorption products on platinum group metals and alloys," Physical Chemistry Chemical Physics, vol. 10, no. 25, pp. 3752-3765, 2008.

[20] T. Bürgi and A. Baiker, "Heterogeneous enantioselective hydrogenation over cinchona alkaloid modified platinum: mechanistic insights into a complex reaction," Accounts of Chemical Research, vol. 37, no. 11, pp. 909-917, 2004.

[21] C. J. Murphy, T. K. Sau, A. M. Gole et al., "Anisotropic metal nanoparticles: synthesis, assembly, and optical applications," Journal of Physical Chemistry B, vol. 109, no. 29, pp. 1385713870, 2005.

[22] C. R. K. Rao and D. C. Trivedi, "Chemical and electrochemical depositions of platinum group metals and their applications," Coordination Chemistry Reviews, vol. 249, no. 5-6, pp. 613631, 2005.

[23] J. R. McBride and M. P. Soriaga, "Adsorbate-catalyzed corrosion. Anodic dissolution of palladium catalyzed by chemisorbed iodine in halide-free acid solutions," Journal of Electroanalytical Chemistry, vol. 303, no. 1-2, pp. 255-259, 1991.

[24] Z. Hu and T. Ritzdorf, "Cyanide- and thiourea-free electrochemical etching of gold for microelectronics applications," Journal of the Electrochemical Society, vol. 154, no. 10, pp. D543-D549, 2007.

[25] G. Baltrūnas, A. Valiūnienè, J. Vienožinskis, E. Gaidamauskas, T. Jankauskas, and Ž. Margarian, "Electrochemical gold deposition from sulfite solution: application for subsequent polyaniline layer formation," Journal of Applied Electrochemistry, vol. 38, no. 11, pp. 1519-1526, 2008.

[26] N. R. Jana, L. Gearheart, S. O. Obare, and C. J. Murphy, "Anisotropic chemical reactivity of gold spheroids and nanorods," Langmuir, vol. 18, no. 3, pp. 922-927, 2002.

[27] H. P. Fong, Y. Wu, Y. Y. Wang, and C. C. Wan, "Electroless Cu deposition process on TiN for ULSI interconnect fabrication 
via $\mathrm{Pd} / \mathrm{Sn}$ colloid activation,” Journal of Electronic Materials, vol. 32, no. 1, pp. 9-17, 2003.

[28] C. D. Iacovangelo and K. P. Zarnoch, "Substrate-catalyzed electroless gold plating," Journal of the Electrochemical Society, vol. 138, no. 4, pp. 983-988, 1991.

[29] S. Hrapovic, Y. Liu, G. Enright, F. Bensebaa, and J. H. T. Luong, "New strategy for preparing thin gold films on modified glass surfaces by electroless deposition," Langmuir, vol. 19, no. 9, pp. 3958-3965, 2003.

[30] G. A. Fried, Y. Zhang, and P. W. Bohn, "Effect of molecular adsorption at the liquid-metal interface on electronic conductivity: the role of surface morphology," Thin Solid Films, vol. 401, no. 1-2, pp. 171-178, 2001.

[31] H. He, W. Cai, Y. Lin, and Z. Dai, "Silver porous nanotube built three-dimensional films with structural tunability based on the nanofiber template-plasma etching strategy," Langmuir, vol. 27, no. 5, pp. 1551-1555, 2011.

[32] S. M. Alia, G. Zhang, D. Kisailus et al., "Porous platinum nanotubes for oxygen reduction and methanol oxidation reactions," Advanced Functional Materials, vol. 20, no. 21, pp. 3742-3746, 2010.

[33] G. H. Jeong, Y. W. Lee, M. Kim, and S. W. Han, "Highyield synthesis of multi-branched gold nanoparticles and their surface-enhanced Raman scattering properties," Journal of Colloid and Interface Science, vol. 329, no. 1, pp. 97-102, 2009.

[34] T. K. Sau and C. J. Murphy, "Room temperature, high-yield synthesis of multiple shapes of gold nanoparticles in aqueous solution," Journal of the American Chemical Society, vol. 126, no. 28, pp. 8648-8649, 2004.

[35] C. H. Cui, H. H. Li, and S. H. Yu, "A general approach to electrochemical deposition of high quality free-standing noble metal (Pd, Pt, Au, Ag) sub-micron tubes composed of nanoparticles in polar aprotic solvent," Chemical Communications, vol. 46, no. 6, pp. 940-942, 2010.

[36] E. Antolini and J. Perez, "The renaissance of unsupported nanostructured catalysts for low-temperature fuel cells: from the size to the shape of metal nanostructures," Journal of Materials Science, vol. 46, no. 13, pp. 4435-4457, 2011.

[37] J. J. Boote and S. D. Evans, "Dielectrophoretic manipulation and electrical characterization of gold nanowires," Nanotechnology, vol. 16, no. 9, pp. 1500-1505, 2005. 

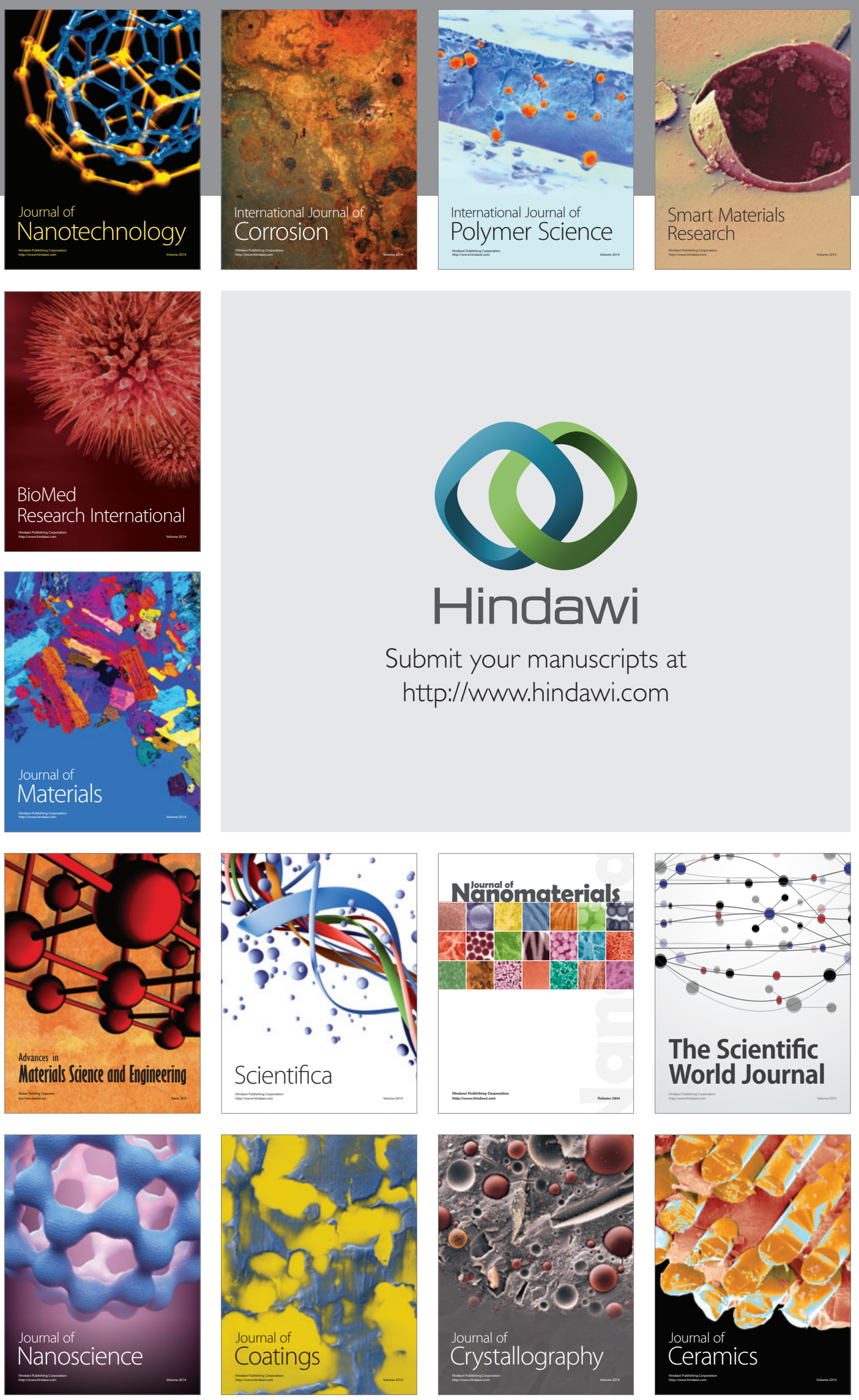

The Scientific World Journal

Submit your manuscripts at

http://www.hindawi.com

\section{World Journal}

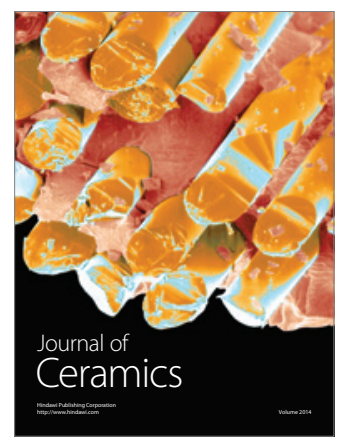

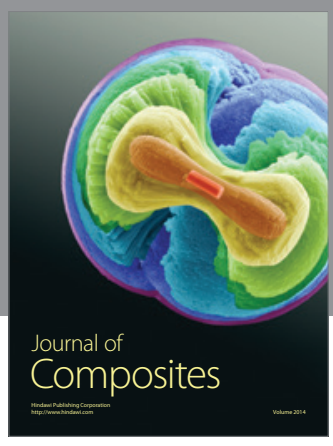
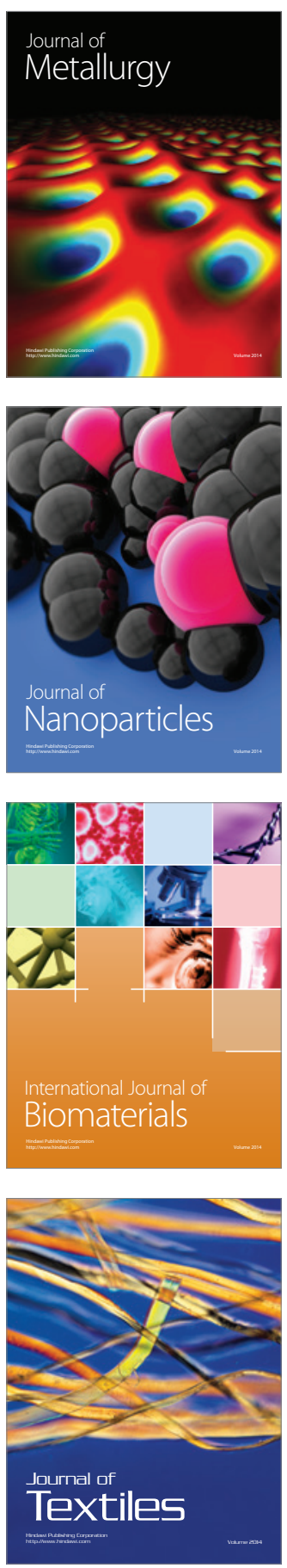